\title{
LASER-INDUCED TRANSIENTS IN COUPLED DOPPLER-BROADENED SYSTEMS $\left({ }^{+}\right)$
}

\author{
M. DUCLOY $\left({ }^{*}\right)$ and M. S. FELD $\left({ }^{+}\right)$ \\ Department of Physics and Spectroscopy Laboratory \\ Massachusetts Institute of Technology \\ Cambridge, Massachusetts 02139, U.S.A.
}

(Reçu le 20 avril 1976, accepté le 3 mai 1976)

\begin{abstract}
Résumé. - Cette lettre propose d'analyser les phénomènes de nutation optique et d'amortissement libre sur une transition 0-2 à l'aide des transitoires induites sur une onde laser résonnante pour une transition couplée $0-1$. Cette technique devrait permettre une analyse directe des phénomènes de relaxation et de déphasage induit par effet Doppler dans un gaz, et fournit une méthode pour distinguer les effets de saturation de populations de ceux dus à des processus de type Raman dans un système à trois niveaux.
\end{abstract}

\begin{abstract}
A new type of experiment is proposed to study optical nutation and free induction decay in a Doppler-broadened transition, by observing the transmission of a weak probe field resonating at a coupled transition. These techniques open new possibilities for direct detailed observations of relaxation phenomena and effects of velocity dephasing in gases, and provide an experimental method for separately studying the effects of population saturation and Raman-type processes in three level systems.
\end{abstract}

When an intense, monochromatic laser field (amplitude $E_{2}$, frequency $\Omega_{2}$ ) resonantly interacts with one of the transitions (0-2, see Fig. 1) of a Doppler broadened atomic or molecular gas, the populations of levels 0 and 2 are altered over a narrow range of axial velocities centered about $v_{2}$, satisfying the resonance condition $\Omega_{2}-k_{2} v_{2}=\omega_{2}$, where $\omega_{2}$ is the center frequency of the 0-2 transition and $k_{2}=\Omega_{2} / c$. This resonant change in the velocity distribution of the level populations manifests itself in the spectral profile of a second Doppler-broadened transition, $0-1$, sharing a common level with $0-2$. If a weak monochromatic field $\left(E_{1}, \Omega_{1}\right)$ collinear with the intense field probes the 0-1 transition (center frequency $\omega_{1}$ )

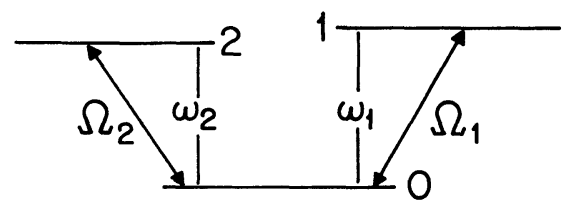

Fig. 1. - Energy-level diagram.

$\left(^{*}\right)$ On leave from Laboratoire de Spectroscopie Hertzienne de l'Ecole Normale Supérieure, Paris, France.

$\left({ }^{+}\right)$Alfred P. Sloan Research Fellow.

${ }^{\dagger}$ ) Work supported in part by Research Corporation and National Science Foundation. a sharp change in transmission occurs when $\Omega_{1}$ is tuned into resonance with the molecules of velocity $v_{2}$ :

$\Omega_{1}=\Omega_{1}(\varepsilon)$, where $\Omega_{1}(\varepsilon)=\omega_{1}+\varepsilon \frac{k_{1}}{k_{2}}\left(\Omega_{2}-\omega_{2}\right)$

$(\varepsilon=+1$ and -1 for copropagating and counterpropagating waves, respectively). This effect, called laser induced line narrowing, has been the subject of numerous theoretical [1] and experimental [2] investigations devoted to studying the steady state change signals at the probe transition [3]. It is now well known that the change signal lineshape cannot be analyzed in terms of population saturation considerations alone, and that double-quantum (Raman-type) processes play an important role. Thus, for example, the widths of the change signals in the forward $(\varepsilon=+1)$ and backward $(\varepsilon=-1)$ directions can differ considerably. This and other lineshape asymmetries have been useful in extracting detailed information about collisional and radiative decay processes in three level systems.

This letter proposes a new type of experiment which studies the transient response at the probe transition when the intense field is suddently turned on (threelevel optical nutation) or off (three-level free decay). These techniques provide an experimental method 
for separating the effects of population saturation and Raman-type processes, and open new possibilities for direct detailed observations of relaxation phenomena and effects of velocity dephasing. Our purpose is to present some of the most striking features of the theoretical predictions and to discuss the new types of information obtainable from transient three-level effects, as compared both to transient two-level effects [4] and to steady state three-level effects.

In the theory the molecular system is described by its density matrix. Going into the rotating frame and making the rotating wave approximation, the highfrequency components are eliminated and the resulting coupled equations describe the behaviour of the slowly varying envelopes, $\sigma_{i j}$, of the density matrix elements. For instance, the equation describing the envelope $\sigma_{01}$ (proportional to the $0-1$ polarization) satisfies [5]

$$
\left[\frac{\mathrm{d}}{\mathrm{d} t}+L_{1}(v)\right] \sigma_{01}=i \beta_{1}\left(\sigma_{11}-\sigma_{00}\right)+i \beta_{2} \sigma_{21}
$$

where $L_{1}=\gamma_{01}+i\left(\Omega_{1}-\omega_{1}-k_{1} v\right) \cdot \gamma_{i j}$ is the relaxation rate of the $\sigma_{i j}$ density matrix element and

$$
\beta_{j}=\mu_{j} E_{j} / 2 \hbar
$$

where $\mu_{j}$ is the $0-j$ electric dipole matrix element. Eq. (1) shows that $\sigma_{01}$ is driven by both the populations of levels 0 and 1 and the $\sigma_{21}$ coherence. The latter coupling is responsible for two photon transitions between levels 2 and 1, a Raman-type process in which the phase of the molecular wave function is not disrupted by the presence of the intermediate state, 0 . The coupled equations can be solved by finding the exact solution for arbitrary $\beta_{2}$ (intense field) when $\beta_{1}$ (weak probe field) is absent, and then using this result to obtain the $\sigma_{i j}$ to first order in $\beta_{1}$. The $\sigma_{i j}$ are then integrated over the velocity distribution. The gain coefficient $G$ of the $0-1$ transition is proportional to $\left\langle\sigma_{01}\right\rangle_{\text {vel }}$.

Let us first consider the three-level free decay signal $\left(\beta_{2} \neq 0\right.$ for $t<0, \beta_{2}=0$ for $\left.t>0\right)$. There is no coupling of $\sigma_{01}$ with $\sigma_{21}$ for $t>0$, and the solution of (1), which is of the form,

$$
\sigma_{01}=A(v) \mathrm{e}^{-\gamma_{0} t}+B(v) \mathrm{e}^{-L_{1}(v) t}+C(v),
$$

exhibits two transients, one $[A(v)$ term $]$ arising from the coupling with $\sigma_{00}$ and the other $[B(v)$ term $]$ describing the decay of $\sigma_{01}$ itself. The decay of $\sigma_{00}$ modifies the stimulated emission (or absorption) at the probe frequency, $\Omega_{1}$. The decay of $\sigma_{01}(t=0)$, in contrast, gives rise to emission at the Dopplershifted natural frequency, $\omega_{1}+k_{1} v$. In the Dopplerbroadened limit the velocity integration of (2) may be performed by closing the integration contour in the lower half of the complex v-plane. Each pole of $A$,
$B$, or $C$ located inside this contour contributes to the integral. As a simple example, consider the free decay signal in the case in which the intense field weakly saturates its transition (i.e. only retain terms up to second order in $\beta_{2} / \gamma$ ), when the transition frequencies are close $\left(\omega_{1} \simeq \omega_{2}\right)$ and dephasing collisions are absent $\left[\gamma_{i j}=\frac{1}{2}\left(\gamma_{i}+\gamma_{j}\right)\right]$. The gain coefficient at the probe transition (for $t \geqslant 0$ ) is given by

$$
\begin{aligned}
\frac{G}{G_{0}}=1-\frac{n_{20}}{n_{10}} & \operatorname{Re}\left[\frac{2 \beta_{2}^{2}}{\gamma(+)+i \delta(\varepsilon)} \times\right. \\
& \left.\times\left(\frac{\mathrm{e}^{-\gamma_{0} t}}{\gamma_{0}}-\frac{1-\varepsilon}{2} \frac{\mathrm{e}^{-[\gamma(-)+i \delta(\varepsilon)] t}}{\gamma(-)+\mathrm{i} \delta(\varepsilon)}\right)\right],
\end{aligned}
$$

where $G_{0}$ is the usual Doppler-broadened 0-1 gain coefficient for $\beta_{2}=0 ; n_{j}$ is the $0-j$ background inversion density; $\delta(\varepsilon)=\Omega_{1}-\Omega_{1}(\varepsilon)$ is the detuning of the probe field from the peak of the change signal; and $\gamma(\varepsilon= \pm 1)$ is the linewidth of the steady state resonance (obtained at $t=0): \gamma(+)=\gamma_{12}$ and $\gamma(-)=\gamma_{01}+\gamma_{02}$. The last term in the brackets describes the decay of the polarization induced by the intense field before $t=0$. Since this polarization is due to molecules in the velocity band centered at $v_{2}=\left(\Omega_{2}-\omega_{2}\right) / k_{2}$, the decay occurs at frequency $\Omega_{1}(\varepsilon)=\omega_{1}+\varepsilon k_{1} v_{2}$. The corresponding emitted field interferes with the probe field to give a beat at frequency $\delta$. In addition to the polarization decay constant, $\gamma_{01}$, the decay of this beat contains a Doppler dephasing contribution, $\gamma_{02}$, due to the velocity spread of the excited molecules, which gives rise to a corresponding spread in the emitted frequencies. The first term in the brackets exhibits neither dephasing contribution nor beat frequency, since it describes a change in stimulated emission resulting from population decay. It is striking that in the forward direction $(\varepsilon=+1)$ there is no contribution from the decay of the induced polarization itself, and thus no Doppler dephasing, contrary to the case of ordinary two-level free decay [4]. This feature is caused by destructive interference in the velocity average of the decaying polarization. There is no such cancellation in the backward direction, and eq. (3) shows that the lineshape of the backward wave is distorted during the decay, contrary to the forward case. Therefore, the forward-backward asymmetry observed in the steadystate change signals also appears in the transient case. Also note that whereas the steady-state signals only give information about the relaxation of the polarizations and level coherence $\left(\gamma_{01}, \gamma_{02}, \gamma_{12}\right)$, the transient signals also directly give the population decay rate $\gamma_{0}$.

For $\omega_{2} \not \omega_{1}$ the transient signal is similar to that of (3) for $\varepsilon=-1$, and also for $\varepsilon=+1$, if $\omega_{2}<\omega_{1}$. When $\omega_{2}>\omega_{1}$ [7] there is an additional contribution to the change signal, eq. (3), due to the decay of a new velocity group prepared in the steady state at $t<0$. This velocity group, associated with the reso- 
nance condition for double quantum processes, is centered at $v_{12}$, defined by

$$
\Omega_{2}-\Omega_{1}-\left(k_{2}-k_{1}\right) v_{12}=\omega_{2}-\omega_{1},
$$

and has a width $\gamma_{12} /\left(k_{2}-k_{1}\right)$. Condition (4) is the statement of energy conservation for the Raman-type process in which a molecule undergoes a transition between levels 2 and 1 by emitting a photon $E_{2}$ and absorbing one at $E_{1}$.

The significance of $v_{12}$ lies in the fact that for $\omega_{2} \neq \omega_{1}$ [7] the two-quantum process is no longer velocity independent, and Raman-type transitions can only occur for molecules in the $v_{12}$ velocity group. The resonant behaviour associated with $v_{12}$ is responsible for the presence of a steady-state change signal for $\varepsilon=+1, \omega_{2}>\omega_{1}$, first observed by Hansch et al. [2], even when the medium is transparent to the saturating field $\left(n_{20}=0, n_{12} \neq 0\right)$. This signal decays according to

$$
\mathrm{e}^{-L_{1}\left(v_{12}\right) t} \propto \mathrm{e}^{-\left\{\gamma_{01}+\left[k_{1} /\left(k_{2}-k_{1}\right)\right] \gamma_{12}+i\left[k_{2} /\left(k_{2}-k_{1}\right)\right] \delta(+)\right\} t},
$$

exhibiting both Doppler dephasing $\left(\gamma_{12}\right.$ term) and distortion of the lineshape.

The optical nutation signal is more complicated than that of free decay because $E_{2}$ is present during the transient. Since now $\beta_{2} \neq 0$ for $t>0, \sigma_{01}$ is coupled directly to $\sigma_{21}$, and indirectly to $\sigma_{02}$ through $\sigma_{00}$ and $\sigma_{21}$. In the limit of weak saturation $\omega_{1} \simeq \omega_{2}$, the forward change signal $(\varepsilon=+1)$ is given by

$$
\begin{aligned}
G / G_{0}= & 1-\frac{n_{20}}{n_{20}} \operatorname{Re}\left[\frac{2 \beta_{2}^{2}}{\gamma(+)-\gamma_{0}+i \delta(+)} \times\right. \\
& \left.\times\left(\frac{1-\mathrm{e}^{-\gamma_{0} t}}{\gamma_{0}}-\frac{1-\mathrm{e}^{-[\gamma(+)+i \delta(+)] t}}{\gamma(+)+i \delta(+)}\right)\right] .
\end{aligned}
$$

As in free decay, this signal is free of Doppler dephasing, but it contains an additional contribution coming from Raman type processes induced by $E_{2}$ during the transient. This contribution decays at a rate $\gamma_{12}$ and oscillates at the driving frequency of the $\sigma_{12}$ coherence, $\delta(+)=\Omega_{1}-\omega_{1}-\Omega_{2}+\omega_{2}$. In the backward direction $(\varepsilon=-1)$ the Raman processes are strongly velocity-dependent, and their net effect vanishes when integrated over velocity. One finds the last term of (6) in brackets is replaced by a Doppler-dephased contribution analogous to the last term of eq. (3) [8] :

$$
2 \frac{1-\mathrm{e}^{-(1 / 2)[\gamma(-)+i \delta(-)] t}}{\gamma(-)+i \delta(-)}
$$

The factor $\frac{1}{2}$ appearing in the exponential gives rise to a decaying oscillation at $\delta(-) / 2$ ! This new type of oscillatory behaviour is a general characteristic of optical nutation transients, but is absent in free decay. Its origin is connected with the appea- rance of a new resonant velocity group, $v^{\prime}$, during the optical nutation transient,

$$
\left(k_{1}+k_{2}\right) v^{\prime}=\Omega_{1}-\omega_{1}-\Omega_{2}+\omega_{2} .
$$

The oscillation at $\delta(-) / 2$ occurs when velocity group $v^{\prime}$ is selected in the component having time behaviour $\mathrm{e}^{-L_{1}(v) t}$. The resonance condition (8) may be interpreted in terms of the quantum process depicted in figure 2 , in which the molecule makes a transition between levels 0 and 2 accompanied by the exchange of three photons with the radiation field,

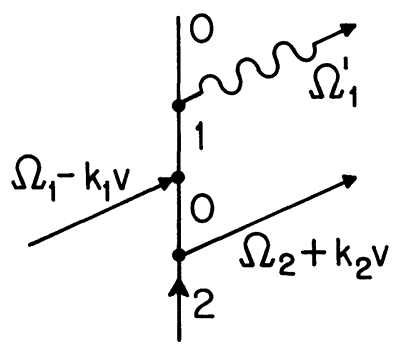

FIG. 2. - Three-quantum process occurring in optical nutation transients. The photon frequencies are given in the molecular rest frame.

emission of a photon $E_{2}$, absorption of a photon $E_{1}$, and emission at the 1-0 transition at a new frequency $\Omega_{1}^{\prime}$ (in the molecular rest frame) determined by the energy conservation condition

$$
\Omega_{1}^{\prime}=\omega_{2}+\Omega_{1}-k_{1} v-\Omega_{2}-k_{2} v .
$$

In the calculation, this emission frequency enters as a decaying component of $\sigma_{01}$ oscillating at $\Omega_{1}^{\prime}(v)$. In the velocity integration this term is resonantly enhanced when $\Omega_{1}^{\prime}$ coincides with the natural frequency $\omega_{1}$, i.e. $v=v^{\prime}$.

In the above discussion phase-changing collisions have been ignored. When such collisions are included $\left[\gamma_{i j}>\left(\gamma_{i}+\gamma_{j}\right) / 2\right]$, Doppler dephasing contributes to the forward signal for both free decay and optical nutation. For instance, the free decay signal contains a contribution similar to the last term of eq. (3). At the limit of strong dephasing collisions (fast $T_{2}$-processes, i.e. $\left.\gamma(-) \gg \gamma_{0}, \gamma(+)\right)$, the term in brackets of eq. (3) becomes :

$$
\begin{aligned}
& 2 \beta_{2}^{2}\left(\frac{\gamma_{0}^{-1}}{\gamma(-)+i \delta(\varepsilon)} \mathrm{e}^{-\gamma_{0} t}+\right. \\
& \left.\quad+\frac{\varepsilon+1}{2} \frac{\gamma(-)^{-1}}{\gamma(+)+i \delta(\varepsilon)} \mathrm{e}^{-\gamma(-) t}\right)^{\prime} .
\end{aligned}
$$

The first term describes a broad resonance [width $\gamma(-)]$ induced by population saturation of level 0 which decays at characteristic rate $\gamma_{0}$. The second term gives the narrow resonance [width $\gamma(+)$ ] due to two-quantum processes, which decays at a much faster rate, $\gamma(-)$, characteristic of the $0-1$ polarization. We obtain the remarkable result that, after a time $\simeq \gamma(-)^{-1}$, the narrow contribution decays away, 
and the forward change signal is reduced to a broad contribution identical to the backward signal (population saturation alone). Thus, in an experiment of this type one can distinguish processes due to population, polarization and double quantum effects. Accordingly, the technique provides a means of separately measuring the decay rates associated with these processes. Similar effects occur in the optical nutation case.

In the case of full saturation $\left(\beta_{2} / \gamma \gg 1\right)$ additional effects such as power broadening and dephasing, dynamic Stark splitting and Rabi-nutation occur. Manuscripts giving a detailed derivation of the complete results are being prepared for publication.
Related analyses have also been given by Brewer et al. [9] and Grischkowsky et al. [10]. Ref. [9] considers the response of a single e.m. field to the sudden splitting of levels 1 and 2 . In this technique, which is limited to forward scattering, one observes a combination of free decay and optical nutation effects [6]. Grischkowsky et al. [10] have analyzed two-photon transient processes in the case of a nonresonant intermediate state. Accordingly, these results are complementary to our own.

We wish to thank Peter Toschek for a stimulating discussion during which this work was conceived, and Jose Leite and Richard Sheffield for useful comments.

\section{References}

[1] Notkin, G. E., Rautian, S. G. and Feoktistov, A. A., $Z h$. Eksp. \& Teor. Fiz. 52 (1967) 1673 [Sov. Phys. JETP 25 (1967)] 1112;

Feld, M. S. and Javan, A., Phys. Rev. 177 (1969) 540 ;

Hansch, T. and ToscheK, P., Z. Phys. 236 (1970) 213;

Feldman, B. J. and Feld, M. S., Phys. Rev. A 5 (1972) 899.

[2] Cordover, R. H., Bonczyk, P. A. and Javan, A., Phys. Rev. Lett. 18 (1967) 730 ;

Holt, H. K., Phys. Rev. Lett. 20 (1968) 410 ;

Hansch, T., KeIl, R., Schabert, A. and ToscheK, P., $Z$. Phys. 226 (1969) 293;

Beterov, I. M. and Chebotayev, V. P., Zh. Eksp. Teor. Fiz. Pis. Red. 9 (1969) 216 [JETP Lett. 9 (1969) 127];

SChabert, A., KeIL, R. and ToscheK, P., Opt. Commun. 13 (1975) 265 and Appl. Phys. 6 (1975) 181.

[3] For a review, see

FELD, M. S., in Fundamental and Applied Physics, edited by M. S. Feld, A. Javan and N. A. Kurnit (Wiley, New York) 1973, pp. 369-420; and

Beterov, I. M. and Chebotaev, V. P., in Progress in Quantum
Electronics 3, edited by J. H. Sanders and S. Stenholm (Pergamon Press, Oxford) 1974.

[4] Hopf, F. A., Shea, R. F. and Scully, M. O., Phys. Rev. A 7 (1973) 2015

Brewer, R. G. and Shoemaker, R. L., Phys. Rev. A 6 (1972) 2001.

[5] A derivation of (1) and of the equations for the other $\sigma_{i j}$ 's may be found in ref. [1] (steady-state) and [6] (transients).

[6] Leite, J., Sheffield, R., Ducloy, M., Sharma, R. and Feld, M., submitted to Phys. Rev.

[7] The precise condition [ref. [3]] is that $\left(\omega_{2}-\omega_{1}\right) \frac{u}{c} \gg \gamma_{12}$, where $u$ is the thermal velocity.

[8] $\delta(+)$ has also to be replaced by $\delta(-)$ in eq. (6).

[9] Shoemaker, R. L. and Brewer, R. G., Phys. Rev. Lett. 28 (1972) 430 ;

Brewer, R. G. and Hahn, E. L., Phys. Rev. A 8 (1973) 464, A 11 (1975) 1641.

[10] Grischkowsky, D., Loy, M. and Liao, P., Phys. Rev. A 12 (1975) 2514. 